Food Diaries to evaluate dietary intake. Nutritional bloods to assess iron and Vitamin D status were taken.

Results Participants included nineteen children. Median age was 7.9 years (range 0.6- 18.1 years). Majority were female $(\mathrm{n}=14,74 \%)$. Median age at diagnosis was 2.5 weeks (range birth - 2.7 years). Growth Hormone treatment was in place for the majority $(n=14,74 \%)$ and commenced at a median age of 2.6 years. Of the reporting parents, $89 \%(n=17)$ were mothers with $37 \% \quad(n=7)$ reporting to be homemakers. All children were living in 2 parent households. BMI was calculated for all children over 2 years $(n=15)$. Using the BMI classification 20\% $(n=3)$ were underweight, 60\% $(n=9)$ were healthy weight, one patient was overweight and 13.3\% $(n=2)$ were obese. Body composition analysis was completed where appropriate $(n=9)$, median $\%$ bodyfat was $26 \%$ and ranged from $10-40 \%$. The majority reported early feeding issues, all of whom required admission to the special care baby unit with median length of stay of 7 days (IQR 14 days). Difficulties progressing with textures and difficulties achieving typical feeding milestones was reported in 7 cases (39\%). Food seeking behaviours were present in 10 patients (55\%) with a median age of onset of 3.7 years. Children achieved 41\% $112 \%$ of their estimated average requirement (EAR) for energy (median 82\%, IQR 33). The macronutrient composition of the diet varied greatly. Insufficient micronutrient intake was reported for iron, calcium and vitamin D. Nutritional bloods identified iron deficiency anaemia and vitamin $\mathrm{D}$ insufficiency in 2 patients. $58 \%(\mathrm{n}=11)$ were taking self-prescribed supplements.

Conclusion Early feeding issues are common in PWS. The majority of our cohort were classified as having a healthy BMI achieved through significant restriction of energy intake. Suboptimal dietary intake of and deficiencies in key nutrients was noted. This study highlights the importance of adjusting energy intake to prevent overweight and obesity while ensuring adequate micronutrient intake to support optimal growth and development.

\section{GP216 AN AUDIT OF MAINTENANCE INTRAVENOUS FLUID THERAPY IN THE PAEDIATRIC GENERAL WARDS AT MATER DEI HOSPITAL, MALTA}

Thomas Calleja*, Jamie Grech, Nikita Taliana, Ray Parascandalo. Department of Child and Adolescent Health, Mater Dei Hospital, Tal-Qroqq, Malta

\subsection{6/archdischild-2019-epa.275}

Background Intravenous fluids are one of the most frequently prescribed drugs in hospital, and yet the practice continues to fall short of National Institute for Health and Care Excellence (NICE) guidelines. Paediatric patients are particularly vulnerable to complications of intravenous fluid therapy.

Aims, objectives and standards We aim to better prescribing practices of intravenous fluid prescription and fluid balance monitoring by staff in the paediatric wards, and to make recommendations and carry out interventions to improve areas where the adherence to NICE guideline 'Intravenous fluid therapy in children and young people in hospital', published in December 2015, is poor, as identified by the first cycle of the audit.

Methodology Data was collected prospectively from the notes of patients aged 0 to 16 years admitted in the two general paediatric wards at Mater Dei Hospital over a four month period. Patients started on intravenous maintenance fluids were included. Data included which fluid was prescribed, the indication, prescription practices, input and output charting and monitoring of serum electrolytes and glucose. Children with diabetic ketoacidosis and renal or hepatic disease were excluded.

Results A total of 65 patients were included. In only 5\% of the treatment charts reviewed was the maintenance intravenous fluid prescribed, despite there being a specific section for fluid prescription. Actual weight was recorded on $89 \%$ of the drug charts. Estimated weight was documented in the remaining $11 \% .81 \%$ of calculations of infusion rate on actual weight were performed correctly according to the HollidaySegar formula. $100 \%$ of patients were administered 5\% dextrose in $0.45 \%$ saline, as per local availabilities. On admission, U\&Es were checked in 97\%, and blood glucose in $79 \%$ of patients. However, U\&Es were only checked in $31 \%$ and blood glucose in $14 \%$ of the patients still on maintenance fluids 24 hours later. Fluid input and output charting was documented in $94 \%$ of patients, but in only $19 \%$ of these patients were subtotals written every 24 hours. The standard charts used at Mater Dei Hospital do not have a section for 12 hourly documentation.

Conclusion Staff within the department must be educated regarding the need for improved intravenous fluid prescription and the importance of conducting daily U\&Es and blood glucose input and output monitoring. Documentation of input and output must also be improved. The input and output charting form needs to be reviewed to include 12 hourly subtotals.

\section{GP217 MARKERS OF ENDOTHELIAL DYSFUNCTION SVCAM-1, VEGF AND METABOLIC STATUS IN OBESE ADOLESCENTS}

${ }^{1}$ Valeria Novikova*, Margarita Gurova ${ }^{1},{ }^{1}$ Olga Gurina, ${ }^{1}$ Olga Varlamova, ${ }^{1}$ Aleksey Blinov, ${ }^{2}$ Irina Burnysheva. ${ }^{1}$ St. Petersburg State Pediatric Medical University, St. Petersburg, Russian Federation; ${ }^{2}$ Children's regional hospital, St. Petersburg, Russian Federation

\subsection{6/archdischild-2019-epa.276}

Introduction Obesity in adolescents is associated with the development of metabolic disorders, chronic inflammation and endothelial dysfunction.

Objectives To determine the peculiarities of metabolic status and presence of markers of endothelial cell dysfunction sVCAM-1 and VEGF-A in adolescents with obesity.

Methods We examined 44 young people: 22 teenagers with obesity (body mass index - BMI - from 30.1 to 42.87) and 22 teenagers with normal physical development (BMI from 18.5 to 24.99). The age of patients ranged from 13 to 18 years (average of $14.25 \pm 1.2$ ). The following laboratory data was screened: level of glucose, insulin, liver enzymes, lipid profile, uric acid, $C$ reactive protein (CRP). Insulin resistance (IR) was estimated by calculation HOMA-IR index. In addition, we analyzed serum concentrations of vascular cell adhesion molecule 1 (sVCAM-1) and vascular endothelial growth factor A VEGF-A - markers that indicate the presence of endothelial dysfunction. Data was analyzed with the use of statistical package Statistica 10.0 for Windows-10. The significance of the differences was determined at $\mathrm{P}$ value $<0.05$.

Results We have identified the following features of carbohydrates and lipid metabolism in obese adolescents: 
hyperinsulinemia in $81.8 \%$, insulinresistancy according to results of HOMA-index - in 72.7\%, increasing triglycerides level - in $40.9 \%$, decreasing level of LPHD - in $77.3 \%$, increasing level of LPLD in $45.6 \%$. CRP was moderately elevated in $31.8 \%$. Liver enzymes (ALT) were increased by $2-3$ times in $40.9 \%$ obese children. Concentration of sVCAM-1 $(1395.23 \pm 264.73 \mathrm{ng} / \mathrm{ml}$ vs $847.44 \pm 190.23 \mathrm{ng} / \mathrm{ml} ; \quad \mathrm{p}<$ $0.0001)$ and VEGF-A $(75.89 \pm 54.79 \mathrm{pg} / \mathrm{ml}$ vs $6.22 \pm 5.74 \mathrm{pg} /$ $\mathrm{ml} ; \mathrm{p}<0.0001)$ was higher in patients with obesity compare to the adolescents with the normal BMI.

Conclusions Obesity in adolescents characterized by significant metabolic disturbances with the development of insulin resistance in $81.8 \%$, atherogenic dyslipidemia in $41 \%$, low-grade inflammation in $31.8 \%$, elevation of liver enzymes in $40.9 \%$ and increased level of endothelial dysfunction markers (sVCAM-1 level in obese teenagers exceeds level of teenagers with normal BMI more than 2 times, VEGF-A - more than 12 times).

\section{GP218 GROWTH PATTERNS IN A PAEDIATRIC OUTPATIENT CLINIC AND ITS ASSOCIATION WITH CHILD EATING BEHAVIOURS AND PARENTAL FEEDING STYLE}

${ }^{1}$ Aisling Lee*, ${ }^{2}$ Louise Rattigan, ${ }^{2}$ Dara Gallagher, ${ }^{2}$ Hilary Greaney, ${ }^{1}$ Laura Keaver. ${ }^{1}$ Institute of technology Sligo, Sligo, Ireland; ${ }^{2}$ Sligo University Hospital, Sligo, Ireland

10.1136/archdischild-2019-epa.277

Background Monitoring the growth pattern of children ensures they are growing at an optimal rate and allows clinical practitioners to detect an over/underweight status. Both can negatively impact a child's health status. In Ireland and in other countries the recognition of childhood obesity outside of medical professionals is poor. Dietary intake is one of the main components that impact a child's weight status. A child's set of eating behaviours is said to be influenced by parental feeding style which in turn impacts on the growth pattern of children.

Aim To determine; (1) the weight status of children aged 2-5 years attending Sligo University Hospital (SUH); (2) if parental feeding style was associated with this weight status and (3) if parents were able to correctly classify their own weight status and their child's and if this was associated with weight status or parental feeding style.

Method A cross-sectional study of children aged 2-5 years and their parents who presented at SUH Paediatric outpatient department between September and November 2018. Anthropometric measures were taken and a demographic and validated parental feeding style questionnaire were completed. Data was analysed using SPSS v24 and significance was set at $\mathrm{P}<0.05$.

Results Thirty-five parents and children were recruited. $80 \%$ of children were of a normal weight status. There was a significant difference between the actual child's weight status and the paternal perception of the child's weight status. Parents who were overweight/obese were statistically more likely to misperceive their child's weight status than parents who were normal weight. There was a trend towards parents who were concerned about their child becoming overweight or obese in the future misclassifying their child's weight status. The most frequently used feeding style in this study was encouragement feeding (80\%). The weight status of the child didn't influence parental feeding style.
Discussion/Conclusion Interestingly, this cohort differs from national rates of childhood obesity. This work supports current literature that parents are poor at recognising an overweight/obese weight status in children aged 2-5 years. Interventions need to be implemented to increase parent's awareness of childhood overweight/obesity and improve their ability to correctly identify these.

\section{GP219 PHYSICAL TRAINING AND NORMOBARIC HYPOXYTHERAPY IN THE REHABILITATION OF OBESE CHILDREN AND ADOLESCENTS}

Aleksandra Mashanskaya, Lyubov Rychkova*, Olga Bugun, Anna Pogodina, Anastasiya Vlasenko. Scientific Centre for Family Health and Human Reproduction Problems, Irkutsk, Russian Federation

10.1136/archdischild-2019-epa.278

The growing prevalence of childhood obesity, which is often associated with other cardiometabolic risk factors (CMR), has become a serious global public health problem. Among modifiable CMR, hypertension occupies a key position. However, there are only a few works on correction of CMR factors in children and adolescents. At the same time, early diagnosis and early management can contribute to rapid reduction of the main manifestations in this cohort.

The Aim To evaluate the effectiveness of combined aerobic exercises and normobaric hypoxytherapy (NHT) in rehabilitation program of adolescents with obesity.

Materials and methods We conducted a comparative study of two groups of overweight/obese adolescents, matched by sex, age, and clinical and biochemical characteristics in combination with hypertension $(\mathrm{n}=72)$. Inclusion criteria: age 13-17 years, overweight (SDS BMI +1.0 to +2.0 ), or obesity (SDS BMI > 2), hypertension, verified by repeated office measurements and 24-hour ambulatory blood pressure monitoring (ABPM). Anthropometric measurements, $24 \mathrm{Hr}$ ABPM, laboratory tests, heart rate variability, psychodiagnostic studies were conducted. Data were processed using statistical software 'Statistica 6.0'. Adolescents in both groups received combined diet and physical exercise therapy. Adolescents of the first group additionally underwent interval hypoxic training (IHT), according to our own method.

Results The inclusion of IHT into the complex therapy had additional benefits in the treatment of adolescents with overweight/obesity and comorbid hypertension. These advantages were characterized by a decrease in cardiovascular reactivity, which was manifested by a decrease in the vagosympathetic interaction index with the formation of significant differences with the control group under orthostatic loading $(\mathrm{p}=0.04)$. The introduction of IHT into the therapeutic complex is largely associated with a lower level of office SBP and DBP after the end of the course of treatment $(p=0.03 ; t=2.2$ for SBP and $p=0.01 ; t=2.6$ for DBP). The positive dynamics of the main components of the functional psychoemotional state was noted when interpreting State-Trait Anxiety Inventory $(p<0.01$ when compared to the control group).

Conclusions An introduction to the complex rehabilitation of adolescents with obesity and arterial hypertension of NHT according to the proposed method will have additional benefits for patients with emotional disorders and increased cardiovascular reactivity. 